\title{
Medicine and the media: the ethics of virtual medical encounters
}

\author{
Authors: Alistair Wardrope ${ }^{\mathrm{A}}$ and Markus Reuber ${ }^{\mathrm{B}, \mathrm{C}}$
}

\begin{abstract}
The expansion of new forms of public media, including social media, exposes clinicians to more illness experiences/narratives than ever before and increases the range of ways to interact with the people depicted. Existing professional regulations and ethics codes offer very limited guidance for such situations. We discuss the ethics of responding to such scenarios through presenting three cases of clinicians encountering television or social media stories involving potential unmet healthcare needs. We offer a structured framework for health workers to think through their responses to such situations, based around four key questions for the clinician to deliberate upon: who is vulnerable to harm; what can be done; who is best placed to do it; and what could go wrong? We illustrate the application of this framework to our three cases.
\end{abstract}

KEYWORDS: Informal medicine, social media, medical professionalism, doctor-patient relationship, clinical ethics, social contract, CRPS, epilepsy

\section{Introduction and background}

The expansion of social media and a persistent appetite for illness narratives in traditional media can place clinicians in inadvertent 'virtual medical encounters' - accounts of real clinical scenarios involving people other than their patients. How should they react when such encounters present them with evidence of apparently unmet clinical needs? What should they do when misinformation could put individuals or the public at risk?

Such virtual encounters are one form of 'informal medicine, the practice of medicine outside an established clinician-patient relationship. Existing attempts to address the ethics of informal medicine highlight the complexities involved in balancing risks to both the person afflicted and the clinician in such scenarios, ${ }^{1,2}$ and advocate evaluating each case on its merits rather than a blanket approach to approving or disapproving of informal medical practice. This complexity is reflected in the lack of firm ethical guidance regarding informal medicine that is provided to

Authors: A core medical trainee, Rotherham NHS Foundation Trust, Rotherham, UK; ${ }^{B}$ professor of neurology, University of Sheffield, Sheffield, UK; Chonorary consultant, Royal Hallamshire Hospital, Sheffield, UK practitioners in professional codes of conduct. Virtual medical encounters, however, pose even more ethical questions than more traditional 'curbside consults', in that they have a greater potential to affect a wide viewing audience. We explore the ethics of virtual medical encounters here by means of three case studies, and outline a framework to help clinicians reason through their responsibilities in such scenarios.

\section{Case studies}

\section{Case 1}

A GP watching a documentary about an emergency department witnesses a patient in a prolonged seizure diagnosed and treated as status epilepticus. He sees that the recorded event is actually a prolonged non-epileptic attack; such misdiagnoses and mistreatment are associated with iatrogenic morbidity and mortality. ${ }^{3}$

\section{Case 2}

A clinician reads a story in an online magazine attributing a sufferer's weakness and sensory impairment to prior administration of the human papillomavirus (HPV) quadrivalent vaccination. She finds no evidence supporting this causal association. $^{4}$

\section{Case 3}

A doctor's friend shares with her a crowd-funding appeal to cover medical costs on social media. The aim is to fund limb amputation for a person with complex regional pain syndrome (CRPS), an intervention not offered through the public health system because it is thought to be futile in most cases and therefore not supported in guidance on CRPS management. ${ }^{5,6}$ Though unlikely to resolve CRPS, the appeal makes it clear that the person believes amputation will be curative.

\section{Approaching the ethics of virtual medical encounters}

What - if anything - puts the clinician who encounters these cases in a morally different position from a layperson? Obviously, there is the difference in medical knowledge. But beyond that, do the professional ethics of healthcare place greater demands on a doctor? 
Existing professional guidance is of limited use in these cases. The General Medical Council (GMC) guidance for doctors on social media concentrates on issues such as privacy, anonymity, and maintaining an appropriate distinction between professional and personal relationships, rather than instances of witnessed medical need. ${ }^{7}$ The British Medical Association (BMA) ${ }^{8}$ and the Royal College of General Practitioners (RCGP) ${ }^{9}$ guidance both briefly address the issue of people soliciting medical advice on social media, but provide little guidance beyond highlighting the risks associated with public provision of personalised medical advice, while still maintaining - in a rather unenlighteningly circular fashion - that 'in situation[s] that require an immediate response' doctors must act 'in the best interests of the patient' and 'follow[ing] your professional obligations as a doctor'. ${ }^{9}$

Thus, explicit professional guidance offers no clear assistance, but nonetheless suggests that clinicians' 'professional obligations' may extend to cover cases like these. Understanding why this should be the case - and what specific responsibilities it might entail - is complicated by the fact that there is no single agreed ethical foundation for doctors' professional responsibilities. Influential approaches to medical ethics such as Beauchamp and Childress' principlism-guided approach to medical decisionmaking explicitly eschew such foundational debates in the hope of providing 'mid-level' principles that may be acceptable to people working from a range of different moral backgrounds. ${ }^{10}$ In a similar vein, we therefore assume that most accounts of professional responsibility would accept the following principle:

Principle A: It is the responsibility of the healthcare professions to promote and protect the health of patients and the public.

Principle A can be justified from a range of perspectives. Most obviously, the 'social contract' model of professional responsibility (endorsed by the Royal College of Physicians, ${ }^{11}$ the American Medical Association, ${ }^{12}$ and the Lancet Medical Professionalism Project $)^{13}$ grounds professionalism in a contract between profession and society, according to which the profession is obliged to act in line with principle A. Alternatively, we may follow Norman Daniels in viewing professional responsibility as arising from considerations of justice. The philosopher John Rawls defines justice as the assurance of fair equality of opportunity - a fair distribution of people's ability to pursue the things that matter most to them. Daniels argues that poor health arbitrarily limits people's range of opportunities, and as such, justice demands that society takes steps to protect people from poor health. The social function of healthcare institutions is to serve this requirement of justice, leading to endorsement of something like principle $A .^{14} \mathrm{~A}$ further potential justification could come from consequentialism (eg utilitarianism) or the principle of beneficence. Both of these positions highlight the ethical importance of the promotion of good outcomes. If we assume that:

1. Health is an important good (either because it is good in itself, or because it is a means to greater human welfare or allowing people to pursue what is most important to them).

2. Given the design of healthcare institutions, and the expertise and training of healthcare professionals, they can best serve the overall good by working to improve health.

Then we arrive at a justification for principle $A$. We discuss the ethical foundations of professional responsibilities and their implications for virtual medical encounters in more detail elsewhere. ${ }^{15}$
Since each case appears to pose some threat to individual or public health, acceptance of principle A should provide sufficient motivation for action. Strictly speaking, however, principle A only establishes that the cases pose problems for which the profession is responsible; it describes a collective responsibility. But where does that leave the individual professional? In normal practice, the profession discharges its collective responsibilities by devolving particular actions to individual members in their particular professional roles, supported by collectively-agreed professional guidance. However, informal medical encounters by definition occur outside the scope of our everyday professional roles, and as we have seen above, existing codes of conduct fail to provide sufficient guidance to the clinician in these cases. Indeed, such codifications would likely be too abstract to engage with the nuances of such cases, or else be unmanageably expansive and unable to keep pace with the shifting landscape of social media. ${ }^{15,16}$

This creates an apparent impasse, where the profession is collectively defaulting on its responsibilities, but no single professional bears any clear individual responsibility. It would be inappropriate to resolve this by making the collective responsibility be the responsibility of every individual clinician - since many challenges to public health cannot be overcome by any individual's actions, this would violate the Kantian (and common sense) dictum that 'ought implies can' - we can only be obligated to perform that which we are able to do. However, if we simply ignored such problems then many potentially ameliorable threats to patient and public health would go unaddressed. We argue elsewhere that this situation should lead clinicians in cases like the above to avoid complacency about their informal responsibilities, and when confronted by problems like these to deliberate seriously on whether, how, and to what extent they may be able to help protect and promote individual or public health. However, given that actions in these situations may be exceedingly difficult, conflict with other personal or professional responsibilities, and by definition occur outside one's normal working role, legal or regulatory obligation to act would prove untenable and encourage potentially damaging regulatory creep out of the clinic and into the home.

Thus we address the ethics of virtual medical encounters, not by prescribing certain courses of action and certainly not by making any claims regarding what conduct regulatory bodies should demand of professionals; we instead suggest some guidance on how to think about the ethics of these situations, and to help clinicians decide whether, how, and to what extent they might be able to act.

\section{Media medical encounters - four questions Responsibilities to whom?}

A first step requires identification of who is vulnerable to harm. Most obviously, this includes the individuals depicted in each case. However, potential vulnerabilities extend beyond these proximate harms: Case 1 provides evidence of clinical error that may be repeated, causing harm to future patients, and that may be symptomatic of a more systemic failing: ${ }^{17}$ Case 3 suggests involvement of a private provider offering a controversial intervention possibly without appraising the patient sufficiently of its risks. There is further potential for diffuse, cumulative impacts on general public health, most apparent in Case 2 which could 
Table 1. Types of vulnerability to harm: individual systemic, and public

Individual

Case 1 Misdiagnosis and mistreatment of a person with NEAD

Case 2 A person's symptoms are attributed erroneously to causation by vaccination; other causes are not explored

Case 3 A person undergoes major surgery with no evidence of likely benefit

\section{Systemic}

Remediable clinical error affecting the treatment of future people presenting with epilepsy/NEAD

Clinicians widely misattribute sets of symptoms to causation by vaccines, preventing future patients accessing appropriate investigation/treatment and reducing their willingness to prescribe vaccinations

Unjustified interventions are offered to other patients with CRPS

\section{Public}

Misinformation about the nature and appropriate treatment of NEAD

Unfounded concerns about side effects produces a vaccine scare, reducing uptake of vaccination amongst at-risk populations

Increased public pressure to offer amputation for CRPS

CRPS = complex regional pain syndrome; $\mathrm{NEAD}=$ non-epileptic attack disorder

fuel another vaccine scare with long-term consequences for cervical cancer prevention. More subtle widespread harms may also arise from Cases 1 and 3. In Case 1, the correct diagnosis of non-epileptic attack disorder (NEAD) is typically delayed by several years, with many patients receiving treatment with antiepileptic drugs with no therapeutic benefit. ${ }^{18}$ The misrepresentation of NEAD as epilepsy in a popular TV documentary may compound diagnostic errors in others and normalise treating NEAD emergencies as status epilepticus. In Case 3 , raising the profile and apparent legitimacy of inappropriate, dangerous surgeries for CRPS could increase pressure on providers to offer such drastic measures. It may help to identify these different levels and types of vulnerability to harm by thinking through in each case: the individual vulnerabilities (what potential or actual harms face those depicted in the scenario); systemic vulnerabilities (to what extent does the scenario demonstrate systematic failings that could put future patients/practitioners at risk); and public vulnerabilities (what harmful effects might arise from widespread dissemination of the misinformation in this scenario?) - see Table 1.

\section{What can be done?}

Potential actions can be roughly divided into four types: They can be local (involving those directly involved in a case) or global (intended to ameliorate/prevent population-level harms); and may be curative (intended to remedy harms) or preventative (stopping similar situations in future) - see Table 2 . Where the bioethics literature or professional guidance addresses informal medicine at all, it concentrates on local and curative actions - the exemplar being 'Good Samaritan' interventions in emergency scenarios. ${ }^{1,2,9}$ However, the situational features that make these the most compelling concerns in Good Samaritan cases - typically the severe, acute nature of the pathology to be addressed, the urgent need for timely intervention, and the lack of other people better positioned to perform such interventions - are not necessarily present in virtual medical encounters like our cases above. Furthermore, the potential to influence more people (by being shared to large online or viewing audiences) means that the cumulative impacts on others may be more significant than the health or the individual depicted in each case.

Table 2. Types of response: local versus global and curative versus preventative

\section{Curative}

Local

Case 1 Correcting the case subject's misdiagnosis of epilepsy and initiating appropriate NEAD management

Case 2 Highlighting likely misattribution of case subject's symptoms to HPV vaccination and reopening differential diagnosis

Case 3 Counselling the case subject regarding other management options for the condition and lack of evidence supporting amputation

\section{Global}

Case 1 Requesting broadcaster highlights potential inaccuracies in medical content of programme to viewing audience

Case 2 Requesting online magazine publishes correction of factual inaccuracies in vaccination story

Case 3 Directly entering discussion on social media, revealing one's professional qualifications and concerns

\section{Preventative}

Highlighting diagnostic and management errors with treating team involved in case; providing relevant education/training

Determining the source of the case subject's beliefs about their condition and correcting misinformation

Approaching the surgical team offering the amputation to discuss the evidence base for such procedure

Developing national/international training on diagnosis and management of patients presenting with transient loss of consciousness

Developing a vaccine education/promotion campaign

Establishing clear guidance from national/ international professional bodies and regular audit procedures on the surgical management of CRPS

CRPS = complex regional pain syndrome; HPV = human papillomavirus; NEAD = non-epileptic attack disorder. 
We therefore recommend breaking down possible actions as depicted in Table 2 to assist in considering the situation from multiple perspectives and thus identifying different potential interventions. Identifying possible actions does not, however, mean they are morally required or even desirable; the next two questions help to decide which (if any) should be pursued.

\section{Who should do it?}

It is the nature of informal encounters that the health worker noticing a situation of potential medical need may not be bestplaced to act upon it. Experts in seizure disorders, infectious diseases or pain medicine will be able to intervene more authoritatively in these cases than a generalist; and both the responsible care team (in the 'local preventative' actions described above) and publishers/broadcasters may be more receptive to interventions from people with relevant expertise. Furthermore, some of the actions described in Table 2 - particularly those falling into the 'global preventative' category - are inherently collective, requiring intervention through training bodies, professional organisations (Cases 1 and 3), or public health authorities (Case 2). Whereas in formal doctor-patient encounters responsibility for managing different aspects of patient or public healthcare are assigned to those with relevant expertise, people finding themselves in an informal encounter may not be experts, and no clear responsibility for action is assigned to someone who might be better placed.

To approach this difficulty, we must recognize that the generalist does not practise in isolation; and just as they may consult with colleagues in their formal practice, so too can they seek advice about informal encounters. The generalist in Case 3 can discuss their concerns with a colleague in pain medicine; this colleague in turn may be able to raise the issue with clinicians formally responsible for the individual's care, who - already knowing the person's more detailed history and having a working relationship with them - are better positioned to discuss concerns regarding surgical intervention. Seeking the expertise of others also begins to create the kind of collective required to stimulate action on the more systemic issues characterized above as global preventative actions.

\section{What could go wrong?}

A last question is whether or how any action may cause harm. Even straightforward curative actions may have damaging results; 'undiagnosing' epilepsy alone (Case 1) may cause considerable distress and affect social networks, social, and financial support. ${ }^{19}$ Potential complications increase as actions involve more people. Heavy-handed intervention involving the surgeon in Case 3 may impose deleterious costs upon health workers only seeking to provide a different approach in the management of a notoriously difficult condition. A vaccination outreach campaign (Case 2) may instead draw attention to a little-known controversy and decrease rates of uptake. Of particular concern is any response that involves making specific, public comments on the diagnosis of an identifiable individual (as would be the likely result of discussing any of these cases in public fora). This (as acknowledged in the 'Goldwater rule') involves breaching norms of confidentiality and consent and risks stigmatising the condition(s) discussed. ${ }^{20}$ These provide a strong presumption against any course of action (such as the 'global curative' response to Case 3 (Table 2)) that would involve public discussion of an identifiable individual's particular case.

Furthermore, 'harm' is not necessarily a univocal concept and people may be benefited in some ways even while being harmed in others, or action to promote one group's interests may harm another. For example, if a person whose online behaviour appears consistent with a mental illness then expresses intent to complete suicide or harm others, a witnessing clinician must weigh the threats to the person's welfare or that of others against the threat to the individual's autonomy of paternalistic intervention. This balancing, however, is not unique to the context of informal medicine or virtual medical encounters; the extensive literature on balancing paternalistic intervention against patient autonomy in other contexts applies here too. ${ }^{10}$

Lastly, there are also potential harms to the person taking action: particularly in the case of controversial diagnoses or treatments, professionals taking an overt stand may attract abuse and even threats of physical harm. Unjustified challenges of another clinician's management could precipitate retaliatory legal or professional action. In all cases, it is therefore vital to consider carefully how any action should be carried out with a minimal risk of inadvertent side effects.

\section{Case analysis}

Applying our approach to each case in turn produces markedly different results. Case 1 is a clear example of misdiagnosis and potentially ongoing mistreatment, but given that he only witnessed one episode, the viewing clinician is not well-positioned to intervene directly; he can, however, attempt to identify a clinician in a more formal clinical relationship with the individual depicted, and raise concerns about training needs with the doctor in charge of the emergency department featured. Local preventative action may be the most pressing response to Case 3 where a surgical team is offering major and unwarranted surgical intervention - though since the surgical team may be aware of further details that make amputation more appropriate, a confidential discussion of concerns would be more measured than open challenge to their proposed management. While all cases highlight potential systemic vulnerabilities that may require global preventative intervention, it is perhaps in Case 2 where that is most apparent. The necessary responses in this case are inevitably collective.

\section{Conclusion}

Encountering stories of apparent medical need via traditional or social media is rapidly becoming a facet of daily life for healthcare workers. Our proposed approach does not provide a clear algorithm for responding to these encounters. Such is their potential variety, and the ethical importance of specific details of each situation, that to attempt this would be a fool's errand. Instead, we have formulated a series of questions to help doctors think through what could and should be done (see Box 1). Rather than give simple answers as to how health workers should act in these scenarios, we hope to have demonstrated a more modest goal; that health care professions - and hence individual professionals - can and should be alert to a potential need to act in situations arising outside their formal practice, and that thinking about who is affected by such cases, what one can do, and how it could go wrong can produce appropriate responses. 
Box 1. Questions to guide reasoning about informal medical encounters

1. Who is vulnerable to harm?

2. What can be done?

a. Consider local and global, curative and preventative courses of action.

3. Who should do it?

a. Consider proximity, expertise, authority and existing therapeutic relationships.

b. If I cannot do anything, can I help or alert someone who can?

4. What could go wrong?

a. Could I harm the case subject, colleagues, the public at large or myself?

b. Are there more or less harmful ways of achieving the same ends?

c. Is there anything I or others can do to mitigate potential side effects?

\section{References}

1 Leavitt FJ, Peleg R, Peleg A. Informal medicine: ethical analysis. J Med Ethics 2005;31:689-92.

2 Mitchell EW. The ethics of passer-by diagnosis. Lancet 2008;371:85-7.

3 LaFrance WC, Benbadis SR. Avoiding the costs of unrecognized psychological nonepileptic seizures. Neurology 2006;66:1620-1.

4 Stillo M, Santisteve PC, Lopalco PL. Safety of human papillomavirus vaccines: a review. Expert Opin Drug Saf 2015;14:697-712.

5 Perez RS, Zollinger PE, Dijkstra PU et al. Evidence based guidelines for complex regional pain syndrome type 1. BMC Neurol 2010:10:20.

6 Bruehl S. Complex regional pain syndrome. BMJ 2015;351:h2730.

7 GMC. Doctors' use of social media. London: GMC, 2013. www.gmc-uk.org/-/media/documents/doctors-use-of-social-media_ pdf-58833100.pdf [Accessed 16 September 2018].
8 British Medical Association. Social media, ethics, and professionalism. London: BMA; 2017.

9 Riley B. RCGP Social Media Highway Code. London: RCGP, 2013. www.rcgp.org.uk/social-media [Accessed 16 September 2018].

10 Beauchamp TL, Childress JF. Principles of biomedical ethics, 7th ed. Oxford: Oxford University Press, 2013:480.

11 Working Party of the Royal College of Physicians. Doctors in society: Medical professionalism in a changing world. London: RCP, 2005. www.rcplondon.ac.uk/publications/doctors-society [Accessed May 9 2014].

12 Wynia MK. The short history and tenuous future of medical professionalism: the erosion of medicine's social contract. Perspect Biol Med 2008;51:565-78.

13 Medical Professionalism Project. Medical professionalism in the new millennium: a physicians' charter. Lancet 2002;359:520-2.

14 Daniels N. Just Health Care. New York: Cambridge University Press, 1985:262.

15 Wardrope A, Reuber M. Diagnosis by documentary: professional responsibilities in informal encounters. Am J Bioeth 2016:16:4050

16 DeMarco JP, Ford PJ. Balancing in ethical deliberation: superior to specification and casuistry. J Med Philos 2006;31:483-97.

17 Leach JP, Lauder R, Nicolson A, Smith DF. Epilepsy in the UK: misdiagnosis, mistreatment, and undertreatment? The Wrexham area epilepsy project. Seizure 2005;14:514-20.

18 Reuber M, Fernandez G, Bauer ] et al. Diagnostic delay in psychogenic nonepileptic seizures. Neurology 2002;58:493-5.

19 Karterud HN, Knizek BL, Nakken KO. Changing the diagnosis from epilepsy to PNES: patients' experiences and understanding of their new diagnosis. Seizure 2010;19:40-6.

20 APA Ethics Committee. American Psychiatric Association Ethics Committee opinion on the Goldwater Rule. APA, 2017. www.psychiatry.org/File \% 20Library/Psychiatrists/Practice/Ethics/ APA-Ethics-Committee-Goldwater-Opinion.pdf [Accessed 11 Dec 2017].

Address for correspondence: Dr Alistair Wardrope, Rotherham Hospital, Moorgate Road, Rotherham S60 2UD, UK.

Email: ajwardrope1@sheffield.ac.uk 